\title{
Old Novae: Old Glories with Bright Future
}

\author{
Alessandro Ederoclite* \\ Centro de Estudios de Física del Cosmos de Aragón \\ E-mail: aederoclecefca.es
}

\section{Claus Tappert}

Instituto de Física y Astronomía, Universidad de Valparaíso

\section{Linda Schmidtobreick}

European Southern Observatory

\author{
Nikolaus Vogt \\ Instituto de Física y Astronomía, Universidad de Valparaíso
}

\begin{abstract}
Classical novae are important astrophysical laboratories and are extremely relevant in several aspects of modern astronomy. A complete understanding of the nova phenomenon passes through the understanding not only of the phase where the nova is bright, but also understanding the properties of the binaries in which these events occur.

This paper focusses on the properties of Cataclysmic Variables (CVs) which have harboured a nova explosion more than 30 years ago. The objects are analysed both in terms of $\mathrm{CV}$ population and in relation to their properties at maximum. Finally, we advice the reader on the proper steps in order to use old novae in the Gaia era.
\end{abstract}

The Golden Age of Cataclysmic Variables and Related Objects IV 11-16 September, 2017

Palermo, Italy

\footnotetext{
*Speaker.
} 


\section{Introduction}

Classical novae are the result of a thermonuclear runaway on the surface of a white dwarf which is accreting mass from a less evolved companion through Roche-lobe overflow. A review can be found in Poggiani [11] or, for a more extensive description, the reader is referred to Bode \& Evans [2] Although novae can be as bright as naked eye objects, they can be very faint when the thermonuclear burning on the white dwarf is finished. For novae discovered in the last two decades the accuracy of their position is already estimated within a few arcseconds while the nova is still at maximum light. This is not true for novae discovered a century or so ago, when the discovery papers often report on the presence of new bright stars close to other known stars but the coordinates are no better than a few arcminutes. This makes their recovery a complicated task.

We normally refer to "old novae" as those novae which are not continuing thermonuclear burning on the surface of the white dwarf and whose light curve has reached quiescence. In other words, an old nova is a cataclysmic variable which harboured a nova explosion and whose brightness is not dominated by the residuals of the thermonuclear runaway.

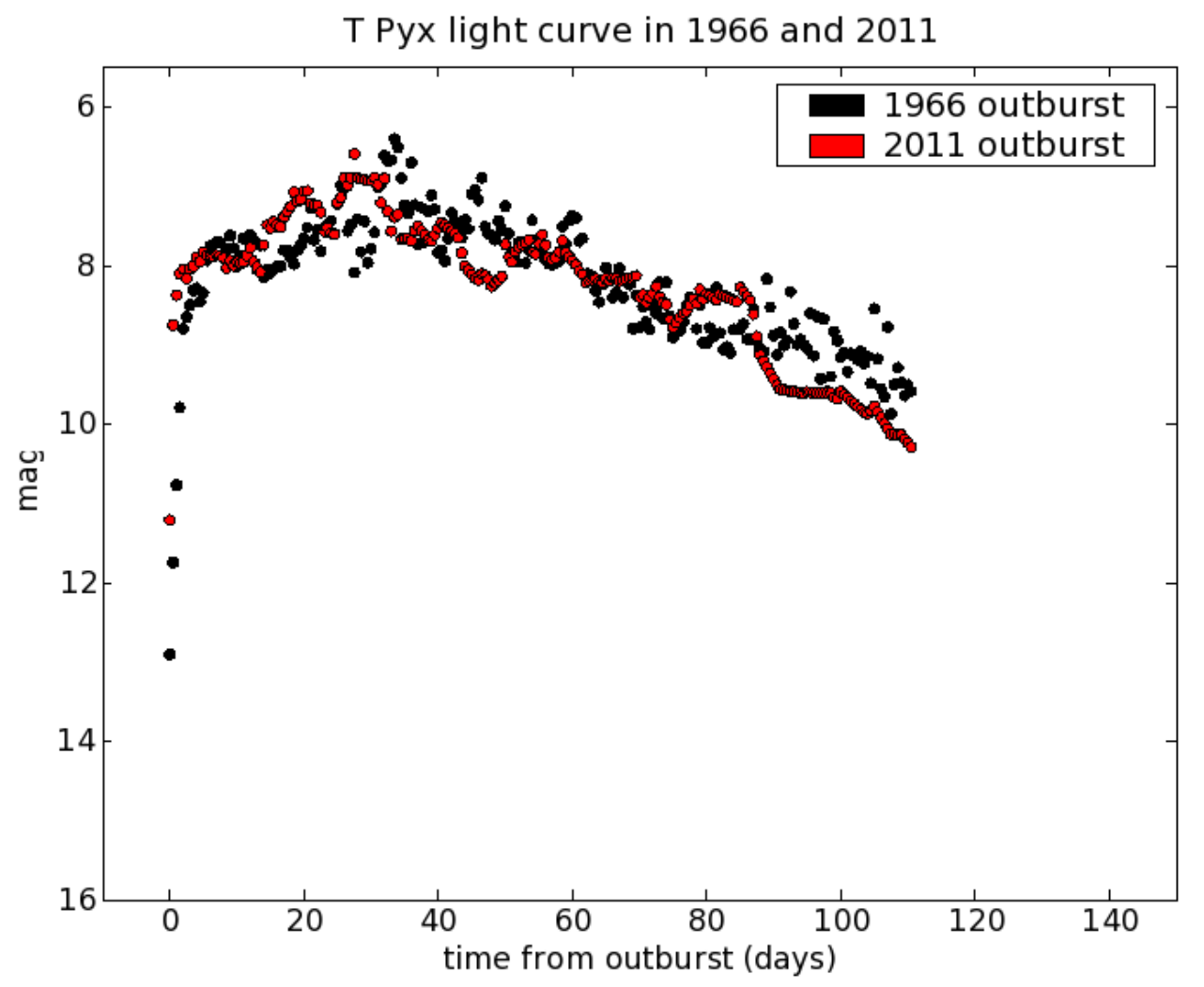

Figure 1: B-band light curve of recurrent nova T Pyx during its last two outbursts (data from AAVSO).

Typically, the time between two nova explosions in the same binary system is of the order of $10^{4}$ years (this time is referred to as "recurrence time"). Recurrent novae are objects in which the recurrence time is as short as a few years or decades and they allow us to study the secular evolution of novae. For example, FIg.1 shows the light curve of the last two explosions of the 
recurrent nova T Pyx. The two light curves are almost indistinguishable, which suggests that two consecutive explosions of a nova may behave very similarly at maximum.

An attempt to describe the long term evolution of CVs after a nova explosion is the so-called "hibernation scenario" proposed by Shara et al. [14]. According to this model, after the nova explosion, the $\mathrm{CV}$ is kept in a high mass-accretion rate by the irradiation of the hot white dwarf. When the white dwarf cools, the secondary star eventually looses contact with its Roche lobe and mass transfer stops, leading the binary to "hibernation". This state lasts several thousands years and accretion is resumed when the secondary comes back in contact with its Roche lobe after loosing angular momentum.

\subsection{Searching for Old Novae}

As mentioned, the search for old novae is a non-trivial task. Tappert et al. ([18], [19], [20], [21], [22], [23]) have carried out a large project aimed at the recovery of these objects, which has resulted in the identification of virtually all the novae older than 30 years and brighter than magnitude 20. The methodology followed by this group is based on the nature of the composite spectrum of CVs. This search is divided in four steps. The first step (in fact a zero-order step) is the search in the literature for information about each nova. The second step is the observation of the field where a nova has occurred. In general, Johnson UBVR images are obtained (yet, in some cases, $\mathrm{H} \alpha$ narrow band imaging proves a highly powerful tool). This allows to probe the shape of the spectral energy distribution of the old nova which is normally identified as an outlier with respect to the position of the field stars in the colour-colour diagram. In fact, the determination is not unique since quasars can resemble CVs (see Abril, Ederoclite and Schmidtobreick [1]), therefore the following step is to measure a spectrum to confirm the nature of the system. The last step is to obtain photometric or spectroscopic time-series to determine the period of the binary. This last step can be time consuming and may require a large telescope (depending on the brightness of the binary) and therefore is not always possible.

It is important to mention that another technique to recover old novae is the direct detection of the expelled material (the "nova shell"). Observationally, this looks like a high density, high expansion velocity planetary nebula, yet in most cases it appears as very faint and diffused since it is observed several centuries after the nova explosion. Shara as well as other authors (Shara et al. [15], Miszalski et al. [9], Shara et al. [16]) have successfully identified a series of shells. In particular, the observation of the shell around Z Cam has been the first evidence of a nova explosion around a long-known low mass-accretion object, thus supporting the "hibernation scenario". Yet, a variety of authors have failed at identifying nova shells around CVs (e.g. Schmidtobreick et al. [13] or Pagnotta \& Zurek [10]). This may be due either to the absence of a nova shell around these objects or to the faintness of the shell.

\subsection{Two Dangerous Concepts}

It is interesting to focus on a concept which is typically taken for granted: the "quiescence" in an old nova. Fig. 2 shows the visible light curve of GK Per (nova Per 1901). A very well studied object, GK Per is known for its dwarf nova outbursts, a behaviour which is inconsistent with a state of "quiescence". In addition, Collazzi et al. [4] report on a decline in brightness of several old novae (the very same GK Per among them) of a few millimagnitudes per year. 


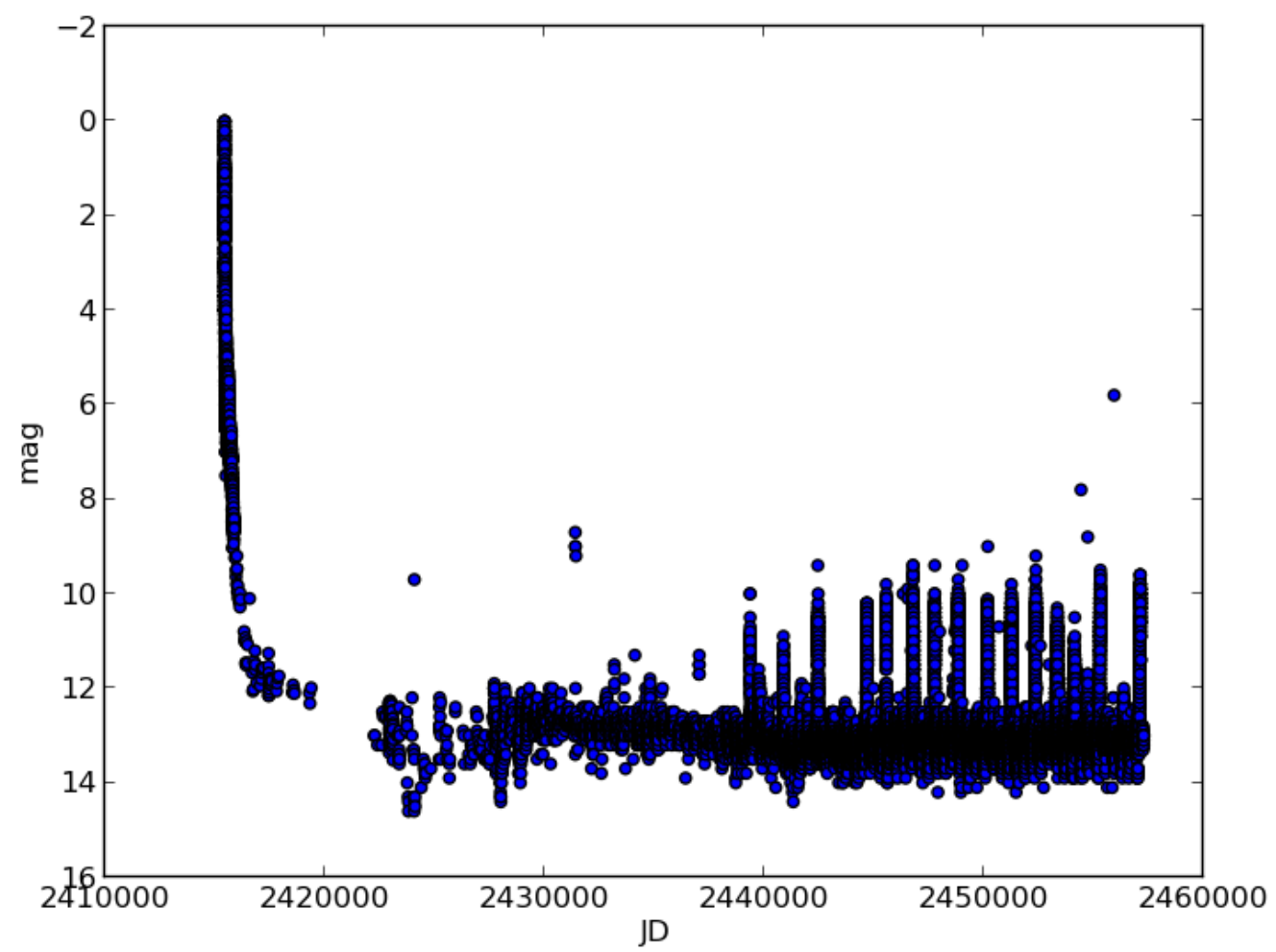

Figure 2: The visual light curve of GK Per as obtained from the AAVSO database.

Even considering the long-term evolution explained in the "hibernation scenario", one could argue that the most quiescent part of the evolution of a $\mathrm{CV}$ is the part which is spent as a detached binary. Yet, the use of this phase is not practical since it occurs on time scales which are too long.

A similarly dangerous concept is the "explosion amplitude" of a nova. It should be emphasized that, when we refer to "explosion amplitude", we are normally referring to the difference between the maximum magnitude and the magnitude of the post-nova. Strictly speaking, there is no reason why the $\mathrm{CV}$ post-nova should have the same brightness of the pre-nova. In fact, considering that a fraction of mass of the system has been expelled from it, one would find reasonable to consider that the pre-nova and the post-nova will have different properties. Nevertheless, since the expelled material is of the order of $10^{-6}-10^{-4} \mathrm{M}_{\odot}$ (see Bode \& Evans [2]), it is likely that the brightness difference between the pre-nova and the post-nova is mostly within the photometric errors, in particular when the object is very faint.

Another important caveat, when referring to the amplitude of the explosion, is that the minimum magnitude of a nova largely depends on the nature of the binary. In fact, if the binary is caught in high mass-accretion phase, it will have a brighter disk and will appear brighter than one caught in a low mass-accretion phase. Even within the same class, the inclination of the disc will have a role in the brightness of the object. A thorough analysis will need to address these issues before drawing firm conclusions. 


\section{The Old Nova Population}

In the following analysis, we focus on 54 objects whose nova explosion occurred more than 30 years ago and for which we could obtain the orbital period either from our observations or from the literature.

Most if not all population studies of CVs focus on the period histogram. In this case, we report in Fig. 3 both the period histogram for the CVs in the Ritter \& Kolb catalogue and the period histogram of old novae. One can see that old novae are mostly distributed above the period gap. In fact, there is a clear peak right at the upper edge of it. The number of old novae below the period gap is very small. This is qualitatively consistent with theoretical modeling by Townsley \& Bildsten [24] and supports the prediction of the "hibernation model" that old novae are in high mass-accretion phase.
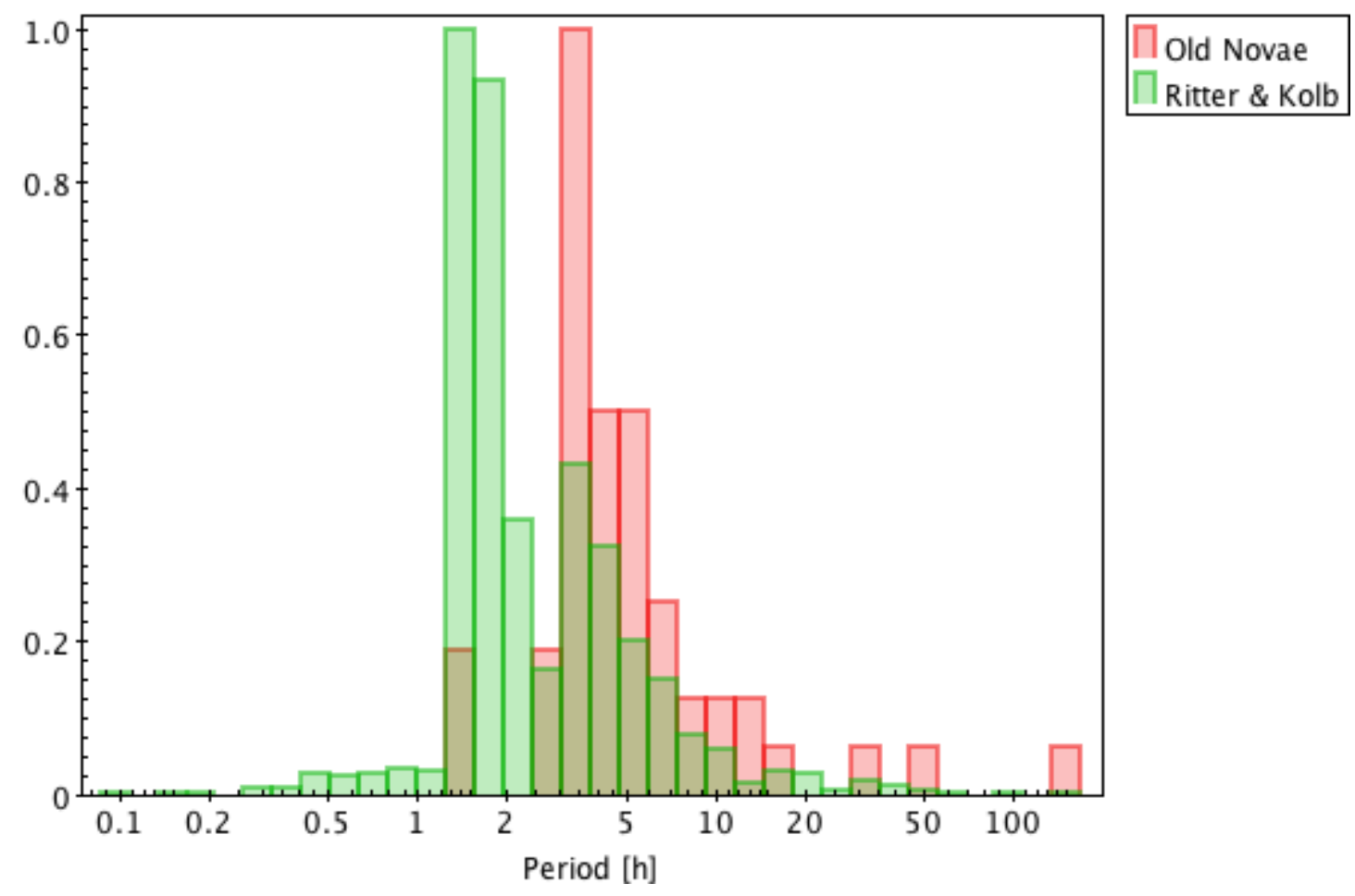

Figure 3: Normalised histogram showing the periods of old novae as compared with all CVs available in the Ritter \& Kolb catalogue.

\section{The Properties at Maximum Brightness of Old Novae}

It is interesting to relate the properties of the old nova with the properties of the nova during maximum. Somehow, it is natural to expect that some properties of the binary (e.g. the mass of the white dwarf) will be related with the properties of the explosion (e.g. the magnitude at maximum).

We begin analysing the speed class and the $t_{n}$, which is the time (usually in days) that a nova needs to become $n$ magnitudes fainter than the maximum light. Most of $t_{n}$ presented here are from Duerbeck [6]. Nevertheless we have re-examined the light curves, whenever possible, with 
AAVSO data and, in some cases, we have updated the value from the literature. As many authors have reported (e.g. Collazzi et al. [4]), novae can have highly irregular light curves, with several rebrighntenings or dips due to the formation of dust. In the present sample, we could determine $t_{2}$ for 15 novae and $t_{3}$ for 41 . One would expect no reason why $t_{2}$ and $t_{3}$ should follow a linear relation. Yet Fig. 4 shows a clear linear trend and only two noticeable objects clearly deviate from it.
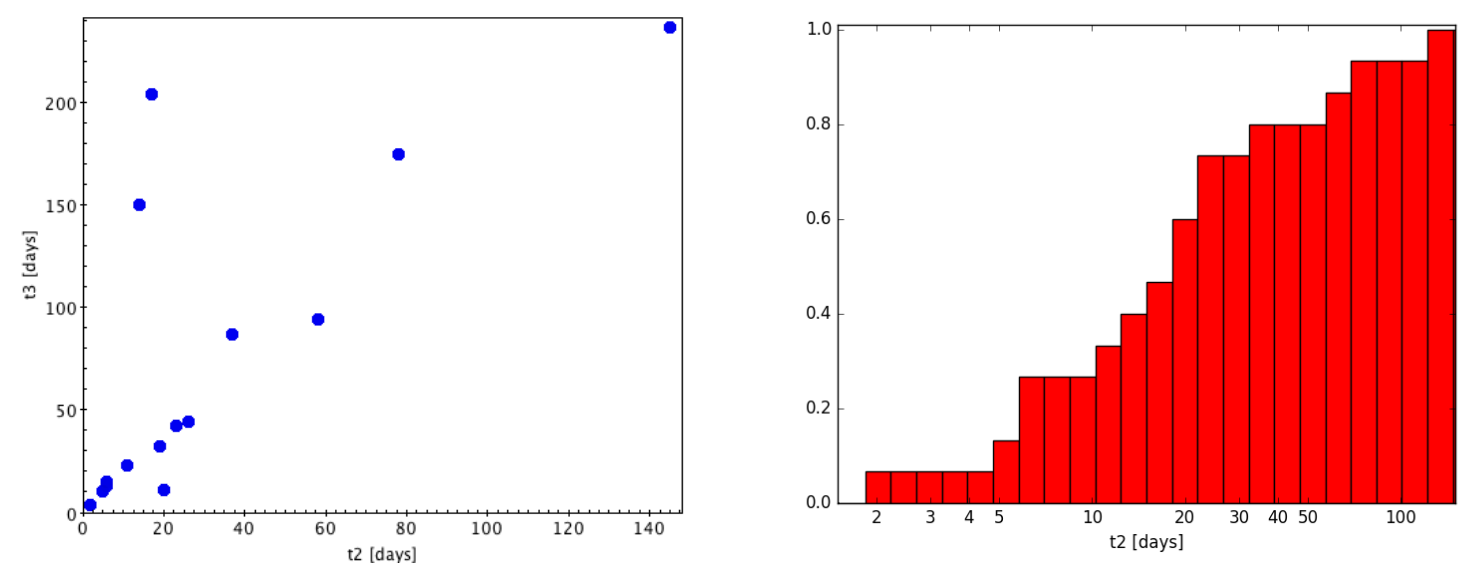

Figure 4: The left panel shows the comparison between $t_{2}$ and $t_{3}$ for our sample. The linear relation is striking. The objects which do not follow the trend are HR Del and RR Pic. The right panel shows the normalised cumulative histogram of $\mathrm{t}_{2}$.

Several authors have used different definitions of "speed class". It is out of the scope of this paper to discuss a new classification based on this parameter. It is enough to mention that, looking at the right panel of Fig. 4 one can tell that less than $30 \%$ of the novae in this sample are fast $\left(t_{2}<12\right.$ days). It becomes $20 \%$ if one defines "fast" as $t_{3}<23$ days. These are important parameters to keep in mind at the moment of designing projects aimed at the discovery of new novae (both Galactic and extra-galactic) as well as understanding the number of novae which occur in our Galaxy.

A very interesting question to ask is if the spectroscopic characteristics of novae at maximum are somehow related with the properties of the binary which harboured the explosion. Williams [26] classified nova spectra as "Fe II" or "He/N" depending on the presence of some lines. In general terms, "Fe II" novae show P-Cyg profiles and smaller ejection velocities with respect to "He/N". It is also assumed that the former occur in systems which have less massive white dwarfs.

In the present work we analyse if the spectral type is related with the amplitude of the explosion and/or the period. Since the Williams classification is, in some cases, several decades more recent than the observation of the novae under investigation, spectroscopic types are derived by the visual analysis of the spectra (whenever published) or by the description of the spectra done in the discovery papers. Only 16 out of the 54 old novae in the present sample could be classified. The left panel of Fig.5 shows the histogram of the explosion amplitude. The number of novae with reliable spectroscopic classification is not enough to draw firm conclusions. In particular, it is worth mentioning the small number of "He/N" novae which can be classified based on the information available. In general terms, the amplitude of a nova explosion is of the order of 10-14 magnitudes. 
This is an important number to keep in mind when searching for systems which harboured a nova explosion, since novae with $m_{\max }>10$ may be beyond the possibility of most telescopes.

The left panel of Fig.6, shows the relation between the amplitude of the explosion and the period of the binary. There seems to be a clear relation between the explosion amplitude and the logarithm of the period. Again, it is hard to draw conclusions based on the difference of the spectroscopic class, given the small number of "He/N" novae. If one assumes that CVs with shorter periods have also lower mass-accretion rate, and, therefore, fainter accretion discs, this relation is qualitatively justified.
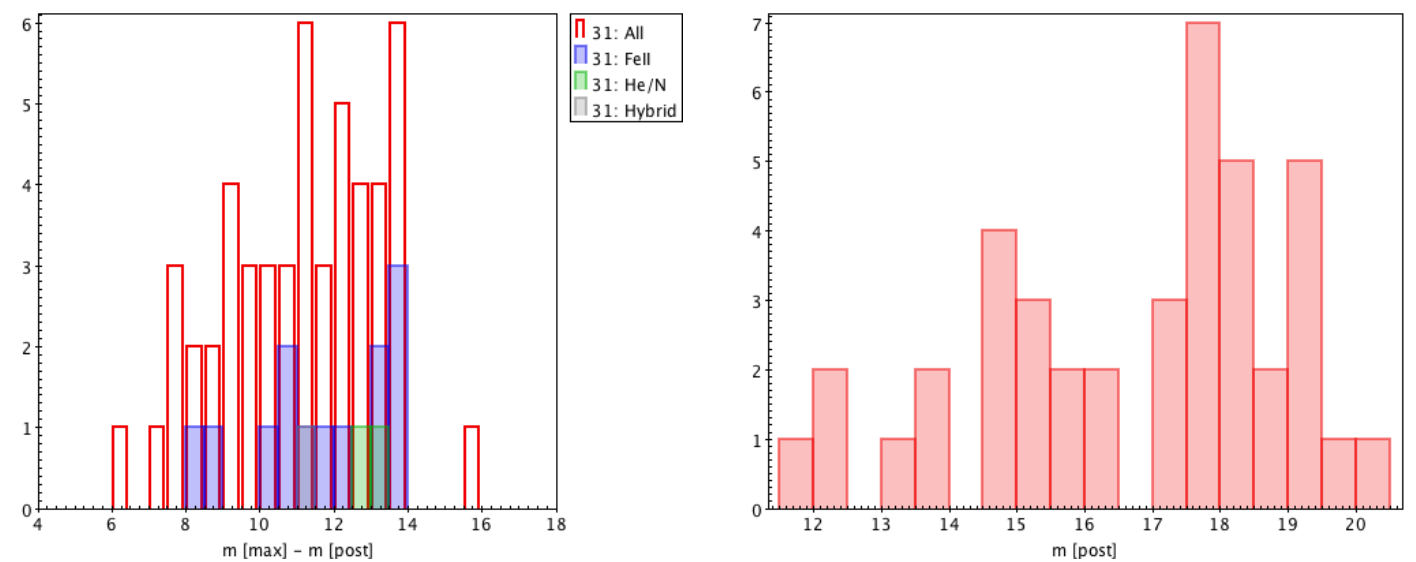

Figure 5: The left panel shows the histogram of the explosion amplitude depending on the spectral type (the colour code refers to the spectral type, when available). The right panel shows the histogram of the brightness of the old novae.
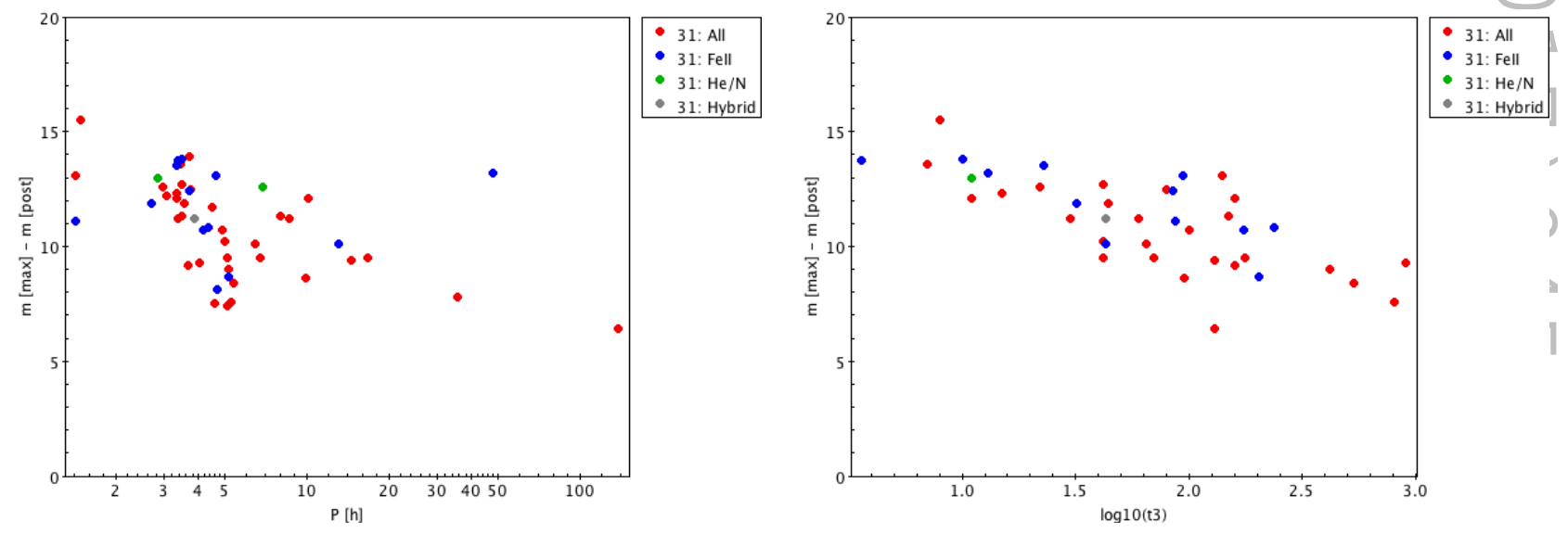

Figure 6: The left panel shows the explosion amplitude as function of the orbital period of the post-nova while the right panel shows the explosion amplitude as function of $t_{3}$ (the colour code refers to the spectral type, when available).

Finally, the right panel of Fig.6 shows the relation between the explosion amplitude and $t_{3}$. In this case, $t_{3}$ is preferred over $t_{2}$ because, as mentioned, there are more novae in this sample with a determination of the former parameter. As already found by Vogt [25], there is a linear relation between the amplitude of the explosion and $t_{3}$. In general terms (see Williams [26] and Della Valle 
[5]), He/N novae are associated with fast novae, while Fe II novae are slower. As for the previous plots, given the small number of objects, it is difficult to support this statement with the data of the current work.

Again, we would like to draw the attention of the reader to the fact that the explosion amplitude is a parameter which must be handled with care for a more complete and detailed analysis.

\section{Old Novae in the Gaia Era}

For many years, novae have been considered a distance indicator through the so-called "maximum magnitude versus rate of decline relationship" (MMRD; see Della Valle [5] and references therein). Several authors (e.g. Kasliwal [8], Shara [17]) have recently argued against the use of the MMRD. In fact, the existence of underluminous fast novae is an issue to establish an MMRD over the full range of $t_{2}$. Yet, most of the evidence on the faults of the MMRD comes from extragalactic novae while the MMRD is based on both Galactic and extragalactic novae.

A lot of emphasis is given recently to the magnificent results coming from the Gaia mission (Brown et al. [3]), an astrometric space mission with unprecedented precision, able to obtain parallaxes for objects as faint as magnitude 20. The first data release of Gaia contains 1,142,679,769 sources. For 2,057,050 sources, an astrometric solution could be derived thanks to the Tycho catalogue, hence this sample is referred to as the "Tycho Gaia Astrometric Solution" (TGAS) and it contains the best parallaxes for these stars to date. A first attempt to use Gaia distances for cataclysmic variables can be found in [12].

The definition of the MMRD based on Galactic novae relies on samples of distances, most of them expansion parallaxes. The Gaia mission will provide precise distances to all the novae which have been used to derive the MMRD and many more. Nevertheless, it is important to proceed with care. As widely known, the absolute magnitude of an object is given by:

$$
M_{\max }=m_{\max }+5-5 \log (d)+A
$$

where $m_{\max }$, the apparent magnitude at maximum can be derived by the observed light curve and $d$, the distance, can be obtained by the Gaia data. It is important to stress that $A$, the interstellar absorption, is the term of the equation which has the largest uncertainty. While some old novae could be observed in ultraviolet, therefore allowing a good determination of the interstellar reddening, this is often not the case, as binaries end up being too faint for spectroscopy on board of the Hubble Space Telescope. In some cases, methods based on optical and/or near-infrared data may be usable (see Ederoclite [7] and references therein).

\section{Summary}

In this paper we have reviewed the state of the research on old novae. The recovery of old novae with photometric techniques is giving very good results. Yet, Fig.5 shows that we are reaching the limit (magnitude about 20) which the current instrumentation allows to obtain time-domain information on old novae. More and more, access to 8 and 10m class telescopes is needed for this science case and, at times, it is not sufficient. 
The search for nova shells is going at a much slower pace. The significant amount of nondetections suggests that an improvement of the technique or the understanding of the long term evolution of nova shells is required. Yet the discovery of nova shells suggesting explosions which occurred centuries ago opens an exciting scenario for the long term evolution of novae.

We have focused our attention on a sample containing 54 novae which exploded more than 30 years ago, but only 16 which could be spectroscopically classified. $30 \%$ of the novae are fast ( $t_{2}<12$ days) and the amplitude of the explosion is mostly between 10 and 14 magnitudes. Although some qualitative relations can be inferred, we stress that some concepts like "quiescence" and "explosion amplitude", which are highly important in this case, should be used with great care.

The sample of spectroscopically characterised old novae is continuously increasing. The most recent novae which are reaching the "30 years" age limit have better data and soon the very same novae used to create the spectroscopic classification will enter the present analysis, providing very interesting and exciting insights.

Finally, in order to take advantage correctly of the data that the Gaia mission will provide it will be crucial to have a good determination of the interstellar extinction. While this is, in principle, feasible using properties of novae at maximum (see Ederoclite [7] and references therein), ultraviolet spectra have proved crucial for "old novae". The limited future of the Hubble Space Telescope will create significant difficulties in the proper exploitation of the data from Gaia. The new ultraviolet missions like the World Space Observatory (WSO) and the Large Ultraviolet-Optical-Infrared Surveyor (LUVOIR) will be highly beneficial, although the latter is only expected in the 2030s.

\section{Acknowledgments}

This work has been supported by the Governments of Spain through the Spanish Ministry of Economy and Competitiveness through the project AYA2015-66211-C2-1-P. The authors wish to thank the American Association of Variable Stars Observers (AAVSO) for making available the photometric data points which have been used to show the light curves used in this paper.

\section{References}

[1] Abril, J., Ederoclite, A., \& Schmidtobreick, L. these proceedings

[2] Bode, M. \& Evans, N. Classical Novae, 2nd Edition, Cambridge University Press, Cambridge, 2008

[3] Brown, A.G.A. et al. 2016, A\&A, 595, 2

[4] Collazzi, A. C. et al. 2009, AJ, 138, 1846C

[5] Della Valle, M. 2002, AIPC, 637, 443

[6] Duerbeck, H. W. 1988, ApL\&C, 27S, 286

[7] Ederoclite, A. et al. 2006, A\&A, 459, 875

[8] Kasliwal, M.M. et al. 2011, ApJ, 735, 94

[9] Miszalski, B. et al. 2016, MNRAS, 456, 633

[10] Pagnotta, A. \& Zurek,D. 2016, MNRAS, 458, 1833

[11] Poggiani, R. these proceedings 
[12] Ramsay, G. et al. 2017, A\&A, 604, 107

[13] Schmidtobreick, L. et al. 2015, MNRAS, 449, 2215

[14] Shara, M. M.et al. 1986, ApJ, 311, 163

[15] Shara, M.M. et al. 2007, Nature, 446, 159

[16] Shara, M.M. et al. 2017, Nature, 548, 558

[17] Shara, M. M. et al. 2017, ApJ, 839, 109

[18] Tappert, C. et al. 2012, MNRAS, 423, 2476

[19] Tappert, C. et al. 2013, MNRAS, 431, 92

[20] Tappert, C. et al. 2013, MNRAS, 436, 2412

[21] Tappert, C. et al. 2014, MNRAS, 442, 565

[22] Tappert, C. et al. 2015, MNRAS, 450, 943

[23] Tappert, C. et al. 2016, MNRAS, 462,1371

[24] Townsley, D. M. \& Bildsten, L. 2005, ApJ, 628, 395

[25] Vogt, N. 1990, ApJ, 356, 609

[26] Williams, R.E. 1992, AJ, 104, 725 